\title{
Special issue on inductive logic programming
}

\author{
Katsumi Inoue $^{1}$ - Hayato Ohwada ${ }^{2}$. \\ Akihiro Yamamoto ${ }^{3}$
}

Received: 20 September 2017 / Accepted: 3 October 2017 / Published online: 14 November 2017

(C) The Author(s) 2017

This special issue focuses on Inductive Logic Programming (ILP). ILP is a subfield of machine learning which uses logic programming as a uniform representation technique, for examples, background knowledge, and hypotheses. Due to its strong representation formalism based on first-order logic, ILP provides excellent means for relational data mining, statistical relational learning, multi-relational data mining, relational reinforcement learning, graph mining, and connections with other learning paradigms. ILP also studies extensions of logic programming languages so that they can be adopted in learning from various types of data.

This special issue followed the 25th International Conference on Inductive Logic Programming, held in Kyoto, Japan (August 20-22, 2015). In our open call for papers, we solicited submissions on ILP. These include theories, such as algorithms, representations, languages, systems, and applications of ILP. We received eight submissions, and we eventually selected four for inclusion in this special issue. The articles have been peer-reviewed according to the journal's high standards.

Every accepted contribution is concerned with foundations of logic programming, learning algorithms, as well as applications, and this is the way of ILP research. Two contributions are mainly focused on learning algorithms and use logic programming as representation, while others are mainly focused on providing logical foundations for application of logic programming to learning. The accepted articles are briefly summarized below.

\footnotetext{
Akihiro Yamamoto

akihiro@i.kyoto-u.ac.jp

Katsumi Inoue inoue@nii.ac.jp

Hayato Ohwada ohwada@rs.tus.ac.jp

1 National Institute of Informatics, Tokyo, Japan

2 Tokyo University of Science, Tokyo, Japan

3 Kyoto University, Tokyo, Japan
} 
In the article "Relational data factorization", Sergey Paramonov, Matthijs van Leeuwen, and Luc De Raedt introduce factorization of relational data in analogy with matrix factorization, one of the most popular methods in machine learning. In matrix factorization, a given matrix is rewritten as the product of other matrices, while in relational data factorization a relation is rewritten as a conjunctive query over other relations, which is the natural join of them. Every new relation is represented by its extension. Because it is known that some ternary relations cannot be rewritten as the join of three binary relations, some constraints and approximation methods must be required. This means that relational data factorization is formalized as an optimization problem for selecting a unique solution from candidate joins under some constraints. To solve the problem answer set programming is applied.

In the article "Planning in Hybrid Relational MDPs" by Davide Nitti, Vaishak Belle, Tinne De Laet, and Luc De Raedt, an algorithm HYPE is designed for probabilistic planning problems involving discrete and continuous states and actions. Markov Decision Processes (MDPs) are popular in modeling probabilistic planning problems, and have been extended in two ways: continuous MDPs for continuous states and actions, and relational MDPs for the case that both states and actions are discrete but represented as objects and relations between them. The latter have been solved with probabilistic logic programming, which is an extension of logic programming so that uncertain facts and rules can be represented in logical formulas and probability. In HYPE dynamic distributed clauses (DDC), which are in a probabilistic logic programming language, are adopted. HYPE is implemented by exploiting the knowledge of the model via importance sampling, which has been used in off-policy Monte Carlo, as well as with overcoming the complexity of regression by sample-based abstraction.

In the article "kProbLog: an algebraic Prolog for machine learning", Francesco Orsini, Paolo Frasconi, and Luc De Raedt introduce an algebraic extension of the logic programming language Prolog. One of the main subjects in ILP is how to recover the lack of expressive power of logic programming languages so that they can be applied to machine learning although various extensions of such languages were proposed. In kProblog facts and rules are annotated by semiring labels to manipulate algebraic expressions in logic, and machine learning techniques such as encoding of graph kernels, expressing declarative formulation of tensor-based algorithms. Theoretical results in semantics of rules, the probabilistic inference, and the guarantee of convergence, as well as practical representation of real world problems are demonstrated.

In first-order logic two types of quantifiers representing "all" and "some" are in the language of formulas. These might be sufficient to represent reasoning in mathematics formally, but other types of quantifiers are required for statistical relational learning where uncertain rules must be induced. In the article "Soft Quantification in Statistical Relational Learning", Golnoosh Farnadi, Stephen H. Bach, Marie-Francine Moens, Lise Getoor, and Martine De Cock introduce a new framework Probabilistic Soft Logic with soft Quantifiers (PSL ${ }^{Q}$ ) for treating soft quantifiers such as for "most" and "a few". The semantics of quantifiers in firstorder logic are provided assuming that a Boolean value 0 or 1 is assigned to each atomic formula without variables, while in $\operatorname{PSL}^{Q}$ the semantics of soft quantifiers are represented by assigning any real number in the interval $[0,1]$ to such an atomic formula. Inference and weight learning methods are also provided for formulas in $\operatorname{PSL}^{Q}$, and some applications to real world problem concerning with networks are demonstrated.

In summary this special issue includes papers demonstrating strong research results in ILP, following both of advances in both machine learning technologies and foundations of logic programming. We hope the readers enjoy them. We would like to thank all the authors and reviewers. Also we extend special thanks to Peter A. Flach, the editor-in-chief of the 
Machine Learning journal and Dragos D. Margineantu, the editor for special issues of the journal.

During the editing period of this special issue, we were all deeply shocked to hear of the premature passing of Dr. Koichi Furukawa. Dr. Furukawa contributed to establish the field of ILP and will be missed by the entire ILP community. In ILP 2015 he was very pleased that ILP conferences were held for 25 years. We would like to dedicate this special issue to the memory of Dr. Furukawa. 